\title{
The Swiss Paediatric Airway Cohort (SPAC)
}

\section{Eva S.L. Pedersen ${ }^{1,9}$, Carmen C.M. de Jong ${ }^{1,9}$, Cristina Ardura-Garcia (1) ${ }^{1}$, Juerg Barben ${ }^{2}$, Carmen Casaulta ${ }^{3}$, Urs Frey ${ }^{4}$, Anja Jochmann ${ }^{4}$, Philipp Latzin (10 ${ }^{3}$, Alexander Moeller ${ }^{5}$, Nicolas Regamey ${ }^{6}$, Florian Singer (i) $^{3,5}$, Ben Spycher ${ }^{1}$, Oliver Sutter ${ }^{7}$, Myrofora Goutaki $\mathbb{1}^{1,3}$ and Claudia E. Kuehni ${ }^{1,3}$, on behalf of the SPAC study group ${ }^{8}$}

Affiliations: ${ }^{1}$ Institute of Social and Preventive Medicine, University of Bern, Bern, Switzerland. ${ }^{2}$ Division of Paediatric Pulmonology, Children's Hospital, St Gallen, Switzerland. ${ }^{3}$ Paediatric Respiratory Medicine, Children's University Hospital of Bern, University of Bern, Bern, Switzerland. ${ }^{4}$ Dept of Paediatrics, University Children's Hospital, University of Basel, Basel, Switzerland. ${ }^{5}$ Division of Paediatric Pulmonology, University Children's Hospital Zurich, University of Zurich, Zurich, Switzerland. 'Division of Paediatric Pulmonology, Children's Hospital, Lucerne, Switzerland. ${ }^{7}$ Paediatric Practice, Worb, Switzerland. ${ }^{8}$ For a full list of members of the SPAC study group see the Acknowledgements section. ${ }^{9}$ These authors contributed equally.

Correspondence: Claudia E. Kuehni, Institute of Social and Preventive Medicine, University of Bern, Finkenhubelweg 11, 3012 Bern, Switzerland. E-mail: claudia.kuehnidispm.unibe.ch

ABSTRACT Chronic respiratory symptoms, such as cough, wheeze and dyspnoea, are common in children; however, most research has, with the exception of a few large-scale clinical cohort studies, been performed in the general population or in small, highly-selected samples.

The Swiss Paediatric Airway Cohort (SPAC) is a national, prospective clinical cohort of children and adolescents who visit physicians for recurrent conditions, such as wheeze and cough, and exercise-related respiratory problems. The SPAC is an observational study and baseline assessment includes standardised questionnaires for families and data extracted from hospital records, including results of clinically indicated investigations, diagnoses and treatments. Outcomes are assessed through annual questionnaires, monthly symptom reporting via mobile phone and follow-up visits.

The SPAC will address important questions about clinical phenotypes, diagnosis, treatment, and the short- and long-term prognosis of common respiratory problems in children. The cohort currently consists of 347 patients from four major hospitals (Bern, Zurich, Basel and Lucerne), with 70-80 additional patients joining each month. More centres will join and the target sample size is a minimum of 3000 patients.

The SPAC will provide real-life data on children visiting the Swiss healthcare system for common respiratory problems and will provide a research platform for health services research and nested clinical and translational studies.

@ERSpublications

The Swiss Paediatric Airway Cohort (SPAC) is a unique research platform for common respiratory problems in children http://ow.ly/Y1v030lDnji

Cite this article as: Pedersen ESL, de Jong CCM, Ardura-Garcia C, et al. The Swiss Paediatric Airway Cohort (SPAC). ERJ Open Res 2018; 4: 00050-2018 [https://doi.org/10.1183/23120541.000502018].

This article has supplementary material available from openres.ersjournals.com

Received: April 032018 | Accepted after revision: Aug 272018

Copyright $\odot$ ERS 2018. This article is open access and distributed under the terms of the Creative Commons Attribution Non-Commercial Licence 4.0. 


\section{Introduction}

Many children suffer during their childhood from repeated episodes of wheeze, cough, or dyspnoea [1-4]. These symptoms are typical for childhood asthma, but are also seen in other diseases. Childhood asthma is characterised by a range of phenotypes that might reflect distinct aetiologies [5-9]. Symptoms vary over time, with short-term diurnal and seasonal fluctuations and a variable long-term course. Factors determining clinical course are poorly understood and the available prediction tools have limited accuracy [10]. In addition, the diagnosis of asthma is not straightforward and making the distinction from healthy children and children with other underlying problems can be difficult $[11,12]$.

Another common and possibly related respiratory problem in childhood is recurrent or chronic cough (without wheeze), which affects up to $20 \%$ of preschool and schoolchildren [3,13-15]. Chronic cough is a key symptom of asthma and upper respiratory tract allergies, but also occurs as prolonged post-infectious cough after viral or atypical bacterial infections, as psychogenic or habit cough, with a retained foreign body, with gastro-oesophageal reflux, or with persistent bacterial bronchitis. Rarely, it reflects a serious underlying condition such as a lung malformation, cystic fibrosis (CF), primary ciliary dyskinesia or immunodeficiency [16]. Finally, exercise-induced respiratory problems are frequent in children with asthma, but can have other causes such as vocal cord dysfunction, laryngomalacia, or primary hyperventilation [17].

Many studies have investigated the epidemiology of childhood asthma. Most were conducted in the general population, such as the International Study on Asthma and Allergies in Childhood (ISAAC) [18, 19], or in birth cohorts [20-24]. Population-based studies are useful for investigating asthma incidence; however, as most children in birth cohorts are healthy or have mild disease, they are of limited utility for studying phenotypes and long-term course. As such, even large cohorts have limited statistical power for studying long-term outcomes of clinically relevant disease. In addition, population-based studies typically rely on questionnaires and simple measurements such as spirometry and allergy tests.

Several clinical cohorts of children with asthma have been established [25-28]. However, their inclusion criteria emphasise asthma alone (e.g. requiring a diagnosis of suspicion of asthma) and do not fully represent the broad mix of phenotypes seen in healthcare. To our knowledge, no multicentre cohort studies have focused on children with recurrent cough as the sole symptom and children with exercise-related symptoms.

Although abundant diagnostic and clinical data are routinely collected by the healthcare system these are rarely used for research. They are of limited value if collected retrospectively from patient records, but can be very valuable if collected prospectively in a standardised way. The Swiss Paediatric Airway Cohort (SPAC) was set up in 2017 as a large, longitudinal database representative of children and adolescents visiting physicians in Switzerland for recurrent wheeze and cough, and exercise-related respiratory symptoms. The SPAC will constitute a national research platform for studying healthcare provision, phenotypes and prognosis, and a sampling frame for conducting nested studies. This article explains the SPAC's aims and its methods, and outlines how the data can be accessed for future research.

\section{Study objectives}

The SPAC is a multipurpose cohort study of children seeking medical care for common lower respiratory problems (cough, wheeze and dyspnoea). Its objectives are: 1) to describe the spectrum and relative frequency of respiratory problems in children visiting pulmonary outpatient clinics and paediatric practices in Switzerland; 2) to distinguish clinical phenotypes of wheeze and cough, and describe associations with physiological traits and measurements; 3) to investigate long-term trajectories and their determinants, and to develop and improve clinical prediction tools; 4) to document diagnostic practises, treatment strategies and preventive measures used in healthcare in Switzerland; 5) to act as a sampling frame for identifying and recruiting children for specific nested studies.

\section{Methods}

Study design

The SPAC is a national multicentre observational study which is integrated into the routine care given by hospitals and practices. Patients are managed according to local procedures and policies with no interference from the study team. No examinations are performed specifically for the SPAC and baseline information is collected prospectively when patients visit the clinic for the first time. Follow-up data are collected by different means, including: medical data from follow-up appointments, questionnaires sent yearly to the families (parents or children themselves from the age of 16 years) and monthly symptom scores assessed via a responsive web application. The study has no specified endpoint, is hosted at the Institute of Social and Preventive Medicine (ISPM, hereafter called the SPAC data centre) at the University of Bern, Switzerland and is managed in close collaboration with all participating SPAC clinics. 


\section{Study population}

Inclusion criteria and participating centres

Patients are included in the SPAC if they are referred to a paediatric respiratory outpatient clinic (or visit a primary care physician repeatedly) for lower respiratory problems such as wheeze, cough, or dyspnoea, or exercise-related breathing problems. This includes children with concomitant upper respiratory symptoms such as sinusitis, rhinitis or adenoid hyperplasia. Eligible patients must be below 17 years old, resident in Switzerland and able to speak sufficient German or French to answer the questionnaire. Exercise-related breathing problems can be wheeze, cough, chest tightness or dyspnoea triggered by exercise.

Excluded children are those with a prior diagnosis of a severe lung disease, such as CF, primary ciliary dyskinesia, severe heart disease, oncological disease, neuromuscular disease, or severe disability (diseases for which there are specific registries) [29]. The SPAC also excludes children who are referred specifically to perform sleep studies for evaluation of sleep-disordered breathing. Exclusion criteria are deliberately few in order to allow the recruitment of a study population representative of the entire spectrum of common lower respiratory problems in childhood, including pre-school viral wheeze, chronic cough and exercise-related problems.

In the first phase, we aim to recruit children from outpatient clinics at all of the major paediatric hospitals in Switzerland (Basel, Bern, Zurich, Lucerne, St. Gallen, Aarau, Lausanne and Geneva). At a later stage, we will open the study to smaller hospitals and paediatric practices.

\section{Study procedures}

\section{Patient identification and recruitment}

Set in different clinics and practices, SPAC logistics vary slightly between centres because systems for planning, inviting and organising patient visits differ (figure 1). Families receive a SPAC information package containing an invitation letter, study information leaflet, informed consent form and questionnaire either in the letter that invites them to the clinic, or upon arrival at the hospital. In both cases, physicians introduce the study to them, answer questions and collect informed consent forms. Families return the completed questionnaire sent to them ahead of time during their visit to the clinic. In the case where clinics distribute the questionnaire upon arrival, families send it directly to the SPAC study centre using a prepaid return envelope. Families who consent but fail to return the questionnaire are mailed a reminder letter with another questionnaire and a prepaid return envelope after 4 weeks. If families do not respond to the first reminder letter, they receive a second reminder 2 months after the first reminder.

\section{Clinical assessments and collection of data from hospital records}

No tests are performed specifically for the SPAC and examinations such as lung function, allergy, blood and bronchial challenge tests are performed only if clinically indicated or if requested by the referring physician (using the respective standard procedures in the clinics involved). The SPAC research team members visit participating centres at regular intervals. Relevant data are extracted from paper and electronic hospital records and entered into the SPAC database.

\section{Follow-up data}

One year after enrolment in the SPAC, caregivers or patients who are 16 years of age or older will receive a follow-up questionnaire by email, text message (sms), or post (figure 2). This will be repeated yearly. Nonresponders will receive a second copy of the follow-up questionnaire after 4-6 weeks and those who do not respond to the second mailing will be contacted by phone. Provided sufficient funding is available, we will also collect medical data from follow-up visits. Beginning in 2019, we will ask for short monthly updates on symptoms, treatments and healthcare utilisation using a simple smart phone app. This will provide prospective data on respiratory symptoms during the year following the baseline medical visit.

\section{What information is collected}

Information from questionnaires

At baseline, families complete a detailed questionnaire that includes sections on frequency, duration, severity, triggers and history of upper and lower respiratory symptoms, as well as diagnoses, treatments, health behaviours, environmental factors, family history and contact information (table 1).

The follow-up questionnaires are shorter and focus on symptoms, treatments and important risk factors during the past 12 months (table 1). Postal addresses for mailing are obtained from hospital records and updated by contacting community registration offices, a procedure used successfully for other registries and cohort studies in Switzerland [30]. Questionnaires are currently available in German, but will be translated into French. 
Step 1: Patient recruitment

Prior to the consultation

Patients receive the SPAC package:

By post with the invitation

to the outpatient clinic

By hand at the SPAC clinic

The SPAC package contains:

Invitation letter to the study

Study information and consent form

Baseline questionnaire

Prepaid envelope to return the

documents

\section{In the consultation}

Physician informs patients and parents about SPAC and registers consent. The signed informed consent form and study questionnaire may be returned at the consultation or by post

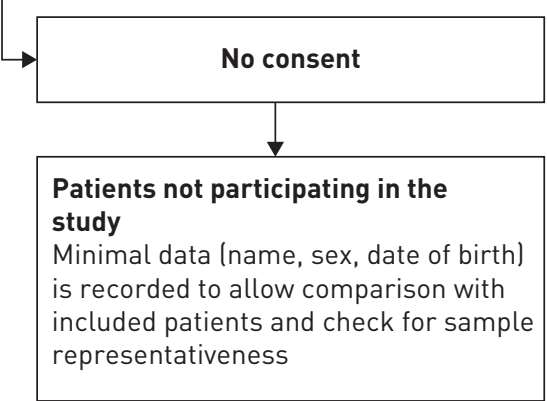

Step 2: Data collection

\section{Baseline}

Personal data

Name, address, date of birth, contact information

Baseline questionnaire

Symptoms, treatment medical history, health behaviours, environment

Medical data (clinic)

Clinical test results, diagnoses, treatment

\section{Follow-up}

Updated personal data

Name, address, date of birth. contact information

Follow-up questionnaire

Symptoms, treatment, health behaviours, environment

\section{Mobile app}

Symptoms, exacerbations, healthcare utilisation, treatment

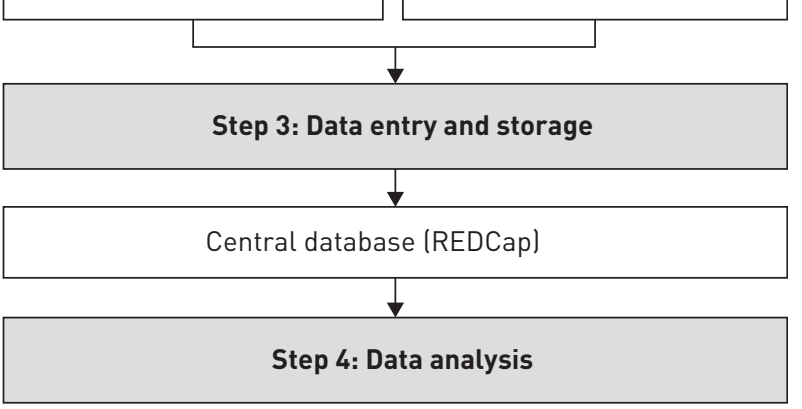

\begin{tabular}{|l|l|l|}
\hline $\begin{array}{l}\text { Identifiable data } \\
\text { Analysed by SPAC data } \\
\text { centre (ISPM) } \\
\begin{array}{l}\text { Pseudo-anonymised data } \\
\text { May be analysed by } \\
\text { collaborators }\end{array}\end{array}$ & $\begin{array}{l}\text { Linking routine data } \\
\text { Swiss national cohort (SNC) } \\
\text { Swiss birth statistics } \\
\text { Hospital episode statistics } \\
\text { Swiss mortality statistics }\end{array}$ \\
\hline
\end{tabular}

FIGURE 1 Schematic chart of data collected in the Swiss Paediatric Airway Cohort (SPAC). REDCap: Research Electronic Data Capture (www. project-redcap.org); ISPM: Institute of Social and Preventive Medicine.

Information from medical records

Information obtained from medical records includes reasons for referral, anthropometric measures, results from physical examinations and diagnostic tests (including pulmonary function tests (PFTs), allergy tests and blood tests), final diagnoses and prescribed treatments (table 1).

Information from the mobile app

From 2019 onwards, parents of participating children and children aged 12 years or older will complete a short questionnaire on symptoms, exacerbations, emergency visits, hospitalisations and use of medication through a simple mobile app. This app will be an adapted version of an existing app called "e-symptoms", developed jointly by the Allergiezentrum Schweiz (www.aha.ch) and CK-Care (www.ck-care.ch) foundations.

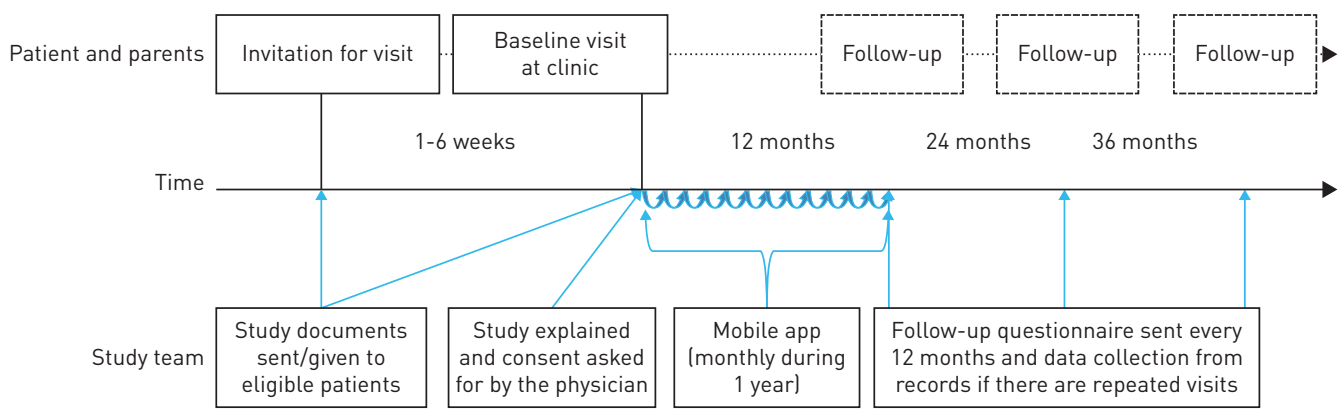

FIGURE 2 Timeline and follow-up procedures of the Swiss Paediatric Airway Cohort. 


\section{Questionnaire data}

Reason for referral

Colds and coughing (frequency, duration, history, severity and triggers)

Wheeze (frequency, duration, history, severity and triggers)

Exercise-related breathing problems (frequency, duration, history, severity and triggers)

Ear, nose and throat (frequency, duration, history and severity of specific conditions, e.g. rhinitis, hay fever and otitis)

Sleeping problems (frequency, duration, history and severity of specific conditions, e.g. sleep apnoea)

Skin (frequency, duration, history, severity and location of specific conditions, e.g. eczema)

Diagnosis and treatment (number of visits to the GP or paediatrician, causes for visiting a physician, tests

performed, medication taken (inhaler medication, oral medication, nasal sprays, eye drops and antibiotics), other chronic illnesses, alternative treatments and vaccinations)

Lifestyle and environment (physical activity, sedentary behaviour, smoking, pets, living on a farm, mould in the house and humidifier use)

Origin and family (citizenship, siblings, parental education and profession, family history of asthma, hay fever and eczemal

Perinatal factors (pregnancy complications, gestational age, birth weight and length, and breastfeeding)

Contact information (address, telephone number and email address)

\section{Data from medical records}

Personal information (date of birth, sex, referring physician and responsible primary care physician)

Disease (diagnoses, dates and results of diagnostic testing, and prescribed treatments)

Measurements ${ }^{\#}$ (weight, height, PFTs (e.g. spirometry and plethysmography), FeNO, spiroergonometry or other exercise challenge tests, bronchial challenge tests (e.g. mannitol and methacholine), allergy tests (total IgE, RAST and SPT), laboratory tests (blood cell count, inflammatory markers and blood gas analysis), imaging (radiography, $\mathrm{CT}$ scan and MRI), microbiology (BAL, sputum and smear), oxygen saturation and $\mathrm{Ptc}_{2}$, bronchoscopy, and special examinations used for differential diagnosis (e.g. chloride sweat test))

\section{Mobile app data}

Symptoms (ear, nose, throat, cough, dyspnoea and wheezing)

Exacerbations (acute worsening of respiratory symptoms)

Healthcare utilisation (hospitalisations and emergency care visits)

Treatment (respiratory symptoms medication)

\section{Routine data and linkage}

SNC lenvironmental exposures (for special analyses) and socioeconomic measures (maternal and paternal education and profession, number of rooms and persons per household, square meter living space per person and area-based socioeconomic position index)l

Swiss birth statistics (gestational age, birth weight and height, and head circumference)

Hospital episode statistics" (type of hospital, length of stay, type of discharge, referral pathways, diagnosis and treatments (ICD-10, procedure and DRGs))

Swiss mortality statistics " (date and cause of death (ICD-10), age, sex, profession, place of residence and citizenship)

$x$

$x$

$x$

$x$

$x$

$x$

$x$

X

X

X

$x$

$x$

$x$

$x$

$x$

GP: general practitioner; PFT: pulmonary function test; FeNO: exhaled nitric oxide fraction; RAST: radio allergo sorbent test; SPT: skin prick test; CT computed tomography; MRI: magnetic resonance imaging; $\mathrm{BAL}$ : bronchoalveolar lavage; $\mathrm{Ptc}_{2}$ : transcutaneous oxygen tension; $\mathrm{SNC}$ : Swiss National Cohort; ICD-10: international classification of diseases-10th revision; DRG: diagnosis related group. \#: only if measurements are taken for clinical reasons; " : birth, hospital episode and mortality statistics will be obtained from the Swiss Federal Statistical Office.

\section{Linkage to routine data}

For specific analyses, we will use probabilistic record linkage to merge data from the SPAC with routine datasets collected by the Swiss Federal Statistical Office, as was done for the Swiss Childhood Cancer Registry (SCCR) [31-33]. This includes information on birth and mortality statistics, national censuses and hospital episode statistics. These data have been linked to build the Swiss National Cohort (SNC), a longitudinal research platform including the entire population of Switzerland [34]. This allows perinatal data, causes of hospitalisations and deaths, socioeconomic indicators and spatially distributed environmental exposures (such as traffic-related air pollution) to be obtained.

\section{Study database}

The SPAC database is web-based, using the Research Electronic Data Capture (REDCap) platform developed at Vanderbilt University (www.project-redcap.org). REDCap is widely used in academic research and allows data entry and extraction in various formats. The REDCap environment is completely secure and only a limited number of research staff from the SPAC research team will have access to the whole dataset. Furthermore, principle investigators at the participating clinics will have access to data from 
only their own clinic. Data entered through the mobile app will be automatically forwarded via a secure connection to a central, secure database accessible only by the study team.

\section{Ethics and data protection}

Ethical approval for the study was obtained from the Bern Cantonal Ethics Committee (Kantonale Ethikkommission Bern 2016-02176). Written informed consent to participate in the study is obtained from patients' parents or directly from patients of 14 years of age or older. Patients can withdraw their consent and their data from the SPAC study at any time by contacting the SPAC clinic or the SPAC data centre. Data generation, transmission and storage, as well as analysis of health-related personal data within the SPAC study, follow the current Swiss legal requirements for data protection. Employees are trained in data protection and must sign an agreement for dealing with particularly sensitive data.

\section{Study power and sample size}

We plan to recruit at least 3000 patients for the SPAC. This number is based on project feasibility and the annual number of patients seen in the paediatric respiratory outpatient clinics in Switzerland. For children with asthma (an estimated $60-70 \%$ of the cohort) this number will allow us to perform analyses such as latent class analyses that involve models with numerous parameters. For developing prognostic models, 10 events are required for each predictor variable entered into the model. A large sample size is also needed to study rarer outcomes such as chronic cough, vocal cord dysfunction and emergency hospital admissions. After reaching 3000 patients, recruitment will continue on a reduced scale, focusing on specific diagnostic subgroups or patients of higher severity.

\section{How data can be accessed}

Participating centres have continual access to their own datasets and can export them directly in various formats for local analyses. The study of SPAC data from several centres is regulated by the SPAC committee, which consists of the SPAC clinics and members of the SPAC data centre. Researchers who wish to use data should submit a concept sheet describing the planned analysis to the SPAC committee for approval (see supplementary material). If the SPAC Committee agrees, a publication agreement (see supplementary material) is signed and the SPAC data centre prepares a partial dataset for the proposed analysis. The SPAC data centre will work closely with and support the lead researchers of each study. Researchers who wish to develop a nested study with inclusion of additional data also need to submit a proposal to the SPAC committee and request permission. Additional data collected by nested studies must be contributed to the SPAC database after the study. Nested studies might also need separate ethics permission.

\section{Current status and initial results}

The first clinics (Lucerne, Bern, Zurich and Basel) began recruiting patients in 2017. During the first months, procedures were revised to fit the requirements of each centre. Currently, 60-80 new patients enter the SPAC every month and enrolment is accelerating (figure 3). St. Gallen and Aarau plan to join in 2018, Lausanne and Geneva in 2019.

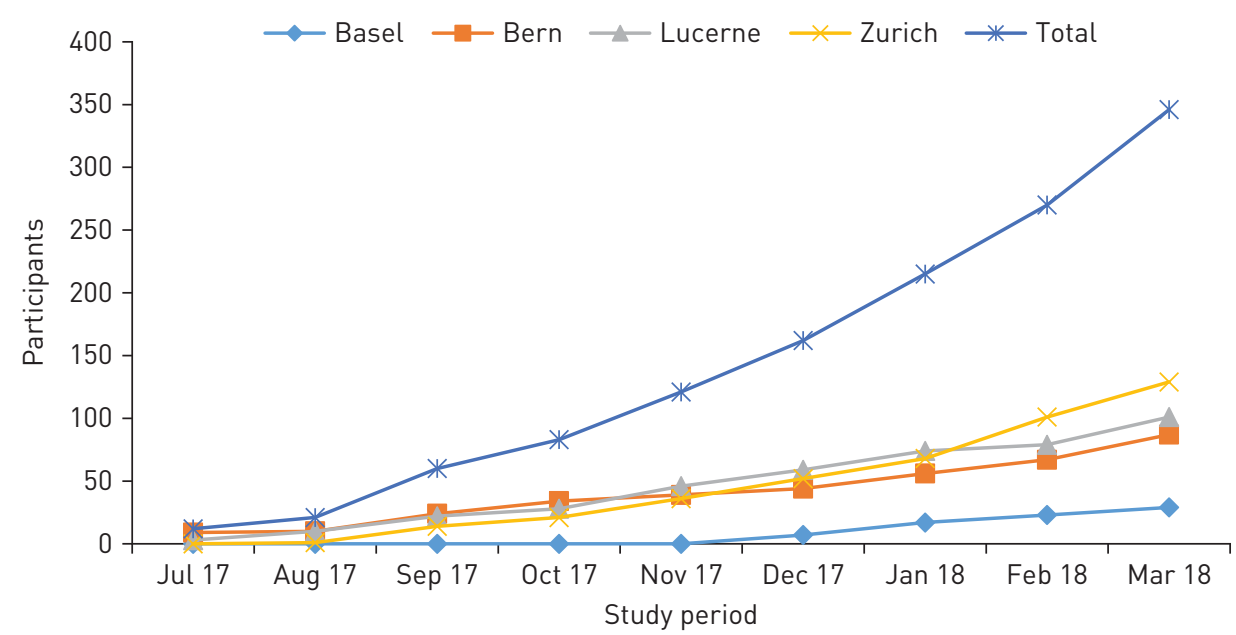

FIGURE 3 Number of participants in the Swiss Paediatric Airway Cohort, from study start in July 2017 to March 2018. 
Until March 2018, 347 out of 851 eligible patients (41\%) consented and returned the baseline questionnaire (table 2). In addition, there were 16 eligible patients who consented but have yet to return the baseline questionnaire. Participation rates varied significantly between hospitals and were $88 \%, 64 \%$, $33 \%$ and 29\%, respectively, in the four centres (Lucerne, Bern, Zurich and Basel), with lower response rates in larger hospitals. Of the patients who participated, $29 \%$ were aged less than 5 years, 33\% were aged 5-9 years and 38\% were aged 10-15 years. Sixty-two percent were males. During the past 12 months, 64\% had wheezed, $50 \%$ had night cough apart from colds, $60 \%$ had exercise-induced symptoms and $13 \%$ had habitual snoring (on most nights). In older children, wheeze and night cough were less common than in younger ones, while exercise-induced symptoms were more common. About $19 \%$ of mothers and $28 \%$ of fathers smoked, $4 \%$ of children lived on a farm and 34\% kept pets at home. During the past 12 months, $75 \%$ had used a short-acting bronchodilator and $56 \%$ had used an inhaled corticosteroid (ICS).

\section{Discussion}

The nationwide and multicentre SPAC is a clinical cohort of children visiting physicians for recurrent wheeze, cough, dyspnoea and exercise-induced respiratory problems. Patients are currently recruited from outpatient clinics of major paediatric hospitals, but smaller hospitals and primary care practices will be invited to join so that the SPAC will include the full spectrum of patients with common respiratory problems in Switzerland. The key features of the SPAC are shown in text box 1.

Only a few comparable clinical cohorts have been set up in other countries. One is the All Age Asthma Cohort (ALLIANCE), a national cohort of paediatric and adult patients with asthma in Germany, led by the German Centre for Lung Research (DZL) and registered at https://clinicaltrials.gov with identifier NCT02496468. ALLIANCE aims to identify biomarkers and predictors of different wheeze phenotypes and their longitudinal course. It differs in several aspects from the SPAC in that it recruits patients with a doctor's diagnosis of asthma and healthy controls, and performs an extensive set of measurements in all

TABLE 2 Characteristics of participants in the Swiss Paediatric Airway Cohort (SPAC) by March 2018

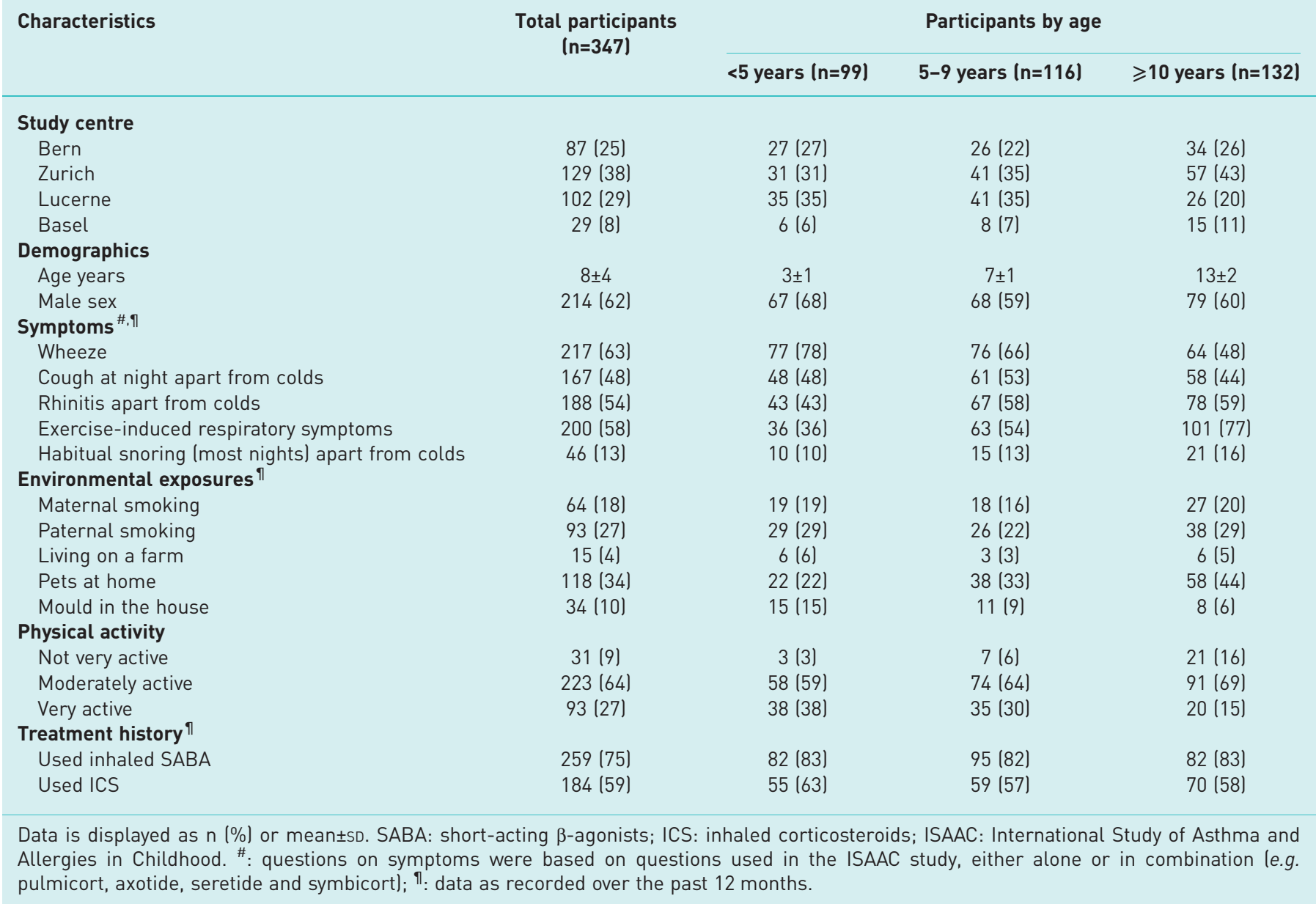


Text Box 1 Key features of the Swiss Paediatric Airway Cohort (SPAC)

- National representative cohort study of children seen in respiratory outpatient clinics.

- Observational study embedded in routine care.

- Focus is on common respiratory problems in childhood (wheeze, cough, exercise- and sleep-related breathing problems).

- Combines patient reported symptoms with data from hospital records.

- Follow-up is via questionnaires and mobile app to families, and linkage to routine data.

- Research platform for nested studies.

children, including single and multiple breath wash-out tests and biomaterial collection (blood, swabs, stool and induced sputum samples) for "omics" studies. Two cohorts from the Netherlands studied preschool children seen in general practice for cough or suspected asthma. One was set up between 1995-1997 [27] and one between 2004-2006 (the ARCADE cohort) [28]. Both followed the children until the age of 6 years and, as in ALLIANCE, these cohorts focused on asthma. They were regional, not nationwide, recruiting participants only from primary care. The Childhood Asthma Management Programme (CAMP) started as a randomised controlled trial (RCT) of over 1000 children aged 5-12 years with asthma who were randomised to receive treatment with budesonide, nedocromil, or a placebo in eight clinical centres in the US and Canada $[25,26]$. Follow-up was extended after the randomisation to study long-term outcomes. As for the other studies mentioned, inclusion criteria were narrow requiring mild to moderate asthma. A strength of this study is that all children received a full set of diagnostic tests and repeated clinical follow-up assessments, including multiple lung function tests.

The SPAC contrasts with all these studies because it is a real-life picture of a representative sample of all children visiting physicians for recurrent wheeze, cough, dyspnoea and exercise problems. Thus it does not only include children with typical doctor-diagnosed asthma, but the entire range of children with less typical features, whose diagnosis is often a challenge for paediatricians and pulmonologists. This setting (consecutive referrals) allows us to assess the value of screening tests, which is not possible in a case-control setting. The SPAC includes not only asthma, but also has a focus on chronic cough and exercise-induced dyspnoea of different aetiologies, both of which are under-researched entities. Being multicentre and observational, the SPAC includes a large proportion of the children referred for evaluation of respiratory disease in Switzerland and allows us to monitor healthcare practise (diagnostics and management) by both referring primary care physicians and hospitals.

This design also leads to weaknesses. First, although inclusion criteria are comprehensive, the study population is not totally representative. Recent immigrants, who do not speak French or German, are not eligible for the study. In addition, the response rate varied between hospitals from $29 \%$ to $88 \%$, suggesting that in some clinics participants are not fully representative of all patients. Furthermore, not all hospitals currently participate and patients seen only in primary care are not currently part of the SPAC. However, despite these limitations, the SPAC represents the mix of patients seen in Switzerland better than a RCT would do, or a prospective study with a fixed set of measurements. Secondly, results from some measurements, such as bronchial challenge tests, will only be available for some patients, and the order and procedures for clinical examinations vary between hospitals. For example, hospitals use different protocols to perform exercise challenge tests. We intend to harmonise these procedures in the future where possible, but the SPAC will remain a real-life observational study. This variation might improve over time because the SPAC provides an opportunity for collaborating centres to harmonise their examinations and the ongoing harmonisation process of the Swiss Personalised Health Network will lead eventually to a more uniform set of data being collected in Swiss hospitals. Thirdly, the lack of sophisticated lung function tests, immunological examinations and biosamples limits the ability of the SPAC to perform deep endophenotyping. Some of these aspects will, however, be covered in nested studies.

In summary, the SPAC will provide a unique resource for studying the pathophysiology, clinical phenotypes and long-term course of common respiratory problems in children, as well as for assessing and comparing aspects of healthcare across Switzerland. This will make it an important research platform for clinical and translational studies on common respiratory problems in children.

Acknowledgements: The paper is presented on behalf of the SPAC study group. We thank all the members of each recruiting centre for their dedicated effort, devotion, promptness and care in the recruitment and assessment of the participants in this study.

Author contributions: C.E. Kuehni, B.D. Spycher, M. Goutaki and the SPAC study group developed the concept and designed the study. C.C.M. De Jong, E.S.L. Pedersen, A. Jochmann, A. Moeller, P. Latzin, C. Casaulta, F. Singer and 
N. Regamey collected the data. C.E. Kuehni, E.S.L. Pedersen, C.C.M. de Jong, C. Ardura-Garcia and M. Goutaki drafted the manuscript. All authors contributed to iterations and approved the final version. C.E. Kuehni takes final responsibility for the contents.

Members of the SPAC study group (www.spac-study.ch): C. Barazzone-Argiroffo, University Children's Hospital, Geneva; J. Barben, Children's Hospital, St Gallen; C. Casaulta, University Children's Hospital, Bern; U. Frey, University Children's Hospital, Basel; A. Jochmann, University Children's Hospital, Basel; P. Latzin, University Children’s Hospital, Bern; A. Moeller, University Children's Hospital, Zurich; N. Regamey, Children's Hospital, Lucerne; F. Singer, University Children's Hospital, Zurich; B.D. Spycher, University of Bern; O. Suter, Paediatric Primary Care, Worb; I. Rochat, University Children's Hospital, Lausanne; and C.E. Kuehni, University of Bern.

Conflict of interest: P. Latzin reports receiving personal fees from Gilead, Novartis, Roche, Schwabe, Vertex, Vifor and Zambon outside the submitted work.

Support statement: The SPAC is funded by the Swiss National Science Foundation (SNF32003B_162820) and several local funding bodies including the Lung League of St Gallen and the Allergiestiftung Ulrich Müller-Gierok. Future grant applications by all partners to different funding bodies will provide continuous support. Funding information for this article has been deposited with the Crossref Funder Registry.

\section{References}

1 Brooke AM, Lambert PC, Burton PR, et al. The natural history of respiratory symptoms in preschool children. Am J Respir Crit Care Med 1995; 152: 1872-1878.

2 Jurca M, Pescatore AM, Goutaki M, et al. Age-related changes in childhood wheezing characteristics: a whole population study. Pediatr Pulmonol 2017; 52: 1250-1259.

3 Jurca M, Ramette A, Dogaru CM, et al. Prevalence of cough throughout childhood: a cohort study. PLoS ONE 2017; 12: e0177485.

4 Martinez FD, Wright AL, Taussig LM, et al. Asthma and wheezing in the first six years of life. The group health medical associates. N Engl J Med 1995; 332: 133-138.

5 Granell R, Henderson AJ, Sterne JA. Associations of wheezing phenotypes with late asthma outcomes in the Avon longitudinal study of parents and children: a population-based birth cohort. J Allergy Clin Immunol 2016; 138: $1060-1070$.

6 Henderson J, Granell R, Sterne J. The search for new asthma phenotypes. Arch Dis Child 2009; 94: 333-336.

7 Just J, Saint Pierre P, Amat F, et al. What lessons can be learned about asthma phenotypes in children from cohort studies? Pediatr Allergy Immunol 2015; 26: 300-305.

8 Spycher BD, Kuehni CE. Asthma phenotypes in childhood: conceptual thoughts on stability and transition. Eur Respir J 2016; 47: 362-365.

9 Spycher BD, Silverman M, Pescatore AM, et al. Comparison of phenotypes of childhood wheeze and cough in 2 independent cohorts. J Allergy Clin Immunol 2013; 132: 1058-1067.

10 Smit HA, Pinart M, Anto JM, et al. Childhood asthma prediction models: a systematic review. Lancet Respir Med 2015; 3: 973-984.

11 Global Initiative for Asthma (GINA). Global strategy for asthma management and prevention, 2017. GINA, 2017. https:/ginasthma.org/wp-content/uploads/2016/01/wms-GINA-2017-main-report-tracked-changes-for-archive.pdf Date last accessed: October 04, 2018.

12 British Thoracic Society (BTS) and the Scottish Intercollegiate Guidelines Network (SIGN). British guideline on the management of asthma: a national clinical guideline. BTS/SIGN, 2016. www.brit-thoracic.org.uk/documentlibrary/clinical-information/asthma/btssign-asthma-guideline-2016/ Date last accessed: October 04, 2018.

13 Brooke AM, Lambert PC, Burton PR, et al. Night cough in a population-based sample of children: characteristics, relation to symptoms and associations with measures of asthma severity. Eur Respir J 1996; 9: 65-71.

14 Brooke AM, Lambert PC, Burton PR, et al. Recurrent cough: natural history and significance in infancy and early childhood. Pediatr Pulmonol 1998; 26: 256-261.

15 Wright AL, Holberg CJ, Morgan WJ, et al. Recurrent cough in childhood and its relation to asthma. Am J Respir Crit Care Med 1996; 153: 1259-1265.

16 Weinberger M, Fischer A. Differential diagnosis of chronic cough in children. Allergy Asthma Proc 2014; 35: 95-103.

17 Abu-Hasan M, Tannous B, Weinberger M. Exercise-induced dyspnea in children and adolescents: if not asthma then what? Ann Allergy Asthma Immunol 2005; 94: 366-371.

18 Pawankar R, Baena-Cagnani CE, Bousquet J, et al. State of world allergy report 2008: allergy and chronic respiratory diseases. World Allergy Organ J 2008; 1: Suppl. 1, S4-S17.

19 Pearce N, Ait-Khaled N, Beasley R, et al. Worldwide trends in the prevalence of asthma symptoms: phase III of the international study of asthma and allergies in childhood (ISAAC). Thorax 2007; 62: 758-766.

20 Bergmann RL, Bergmann KE, Lau-Schadensdorf S, et al. Atopic diseases in infancy. The German multicenter atopy study (MAS-90). Pediatr Allergy Immunol 1994; 5: Suppl. 1, 19-25.

21 Kuehni CE, Brooke AM, Strippoli MP, et al. Cohort profile: the Leicester respiratory cohorts. Int J Epidemiol 2007; 36: 977-985.

22 Simpson BM, Custovic A, Simpson A, et al. NAC Manchester asthma and allergy study (NACMAAS): risk factors for asthma and allergic disorders in adults. Clin Exp Allergy 2001; 31: 391-399.

23 Taussig LM, Wright AL, Morgan WJ, et al. The Tucson children's respiratory study. I. Design and implementation of a prospective study of acute and chronic respiratory illness in children. Am J Epidemiol 1989; 129: 1219-1231.

24 Wijga $\mathrm{AH}$, Kerkhof M, Gehring U, et al. Cohort profile: the prevention and incidence of asthma and mite allergy (PIAMA) birth cohort. Int J Epidemiol 2014; 43: 527-535.

25 Szefler S, Weiss S, Tonascia J, et al. Long-term effects of budesonide or nedocromil in children with asthma N Engl J Med 2000; 343: 1054-1063. 
Covar RA, Fuhlbrigge AL, Williams P, et al.The childhood asthma management program (CAMP): contributions to the understanding of therapy and the natural history of childhood asthma. Curr Respir Care Rep 2012; 1 : 243-250.

27 Eysink PE, ter Riet G, Aalberse RC, et al. Accuracy of specific IgE in the prediction of asthma: development of a scoring formula for general practice. Br J Gen Pract 2005; 55: 125-131.

28 van Wonderen KE, van der Mark LB, Mohrs J, et al. Prediction and treatment of asthma in preschool children at risk: study design and baseline data of a prospective cohort study in general practice (ARCADE). BMC Pulm Med 2009; 9: 13.

29 Goutaki M, Maurer E, Halbeisen FS, et al. The international primary ciliary dyskinesia cohort (iPCD Cohort): methods and first results. Eur Respir J 2017; 49: 1601181.

30 Kuehni CE, Rueegg CS, Michel G, et al. Cohort profile: the Swiss childhood cancer survivor study. Int J Epidemiol 2012; $41: 1553-1564$.

31 Adam M, Rueegg CS, Schmidlin K, et al. Socioeconomic disparities in childhood cancer survival in Switzerland. Int J Cancer 2016; 138: 2856-2866.

32 Schindler M, Spycher BD, Ammann RA, et al. Cause-specific long-term mortality in survivors of childhood cancer in Switzerland: a population-based study. Int J Cancer 2016; 139: 322-333.

33 Spycher BD, Lupatsch JE, Zwahlen M, et al. Background ionizing radiation and the risk of childhood cancer: a census-based nationwide cohort study. Environ Health Perspect 2015; 123: 622-628.

34 Spoerri A, Zwahlen M, Egger M, et al. The Swiss national cohort: a unique database for national and international researchers. Int J Public Health 2010; 55: 239-242. 